\section{ANÁLISIS COMPARATIVO DE LA LÓGICA TÉCNICO-POLÍTICA DE RECONSTRUCCIÓN: TERREMOTO E INCENDIOS FORESTALES EN EL MAULE, CHILE'}

Stefano Micheletti ${ }^{2}$, Dante Pancani ${ }^{3}$,Elena Pisani $^{4}$

\section{Resumen}

Los incendios forestales del 2017 ocasionaron importantes daños en la Región del Maule, y obligaron a un nuevo proceso de reconstrucción en las zonas rurales que ya habían sido afectadas por el terremoto del 2010. Este artículo realiza un análisis comparativo de la lógica técnico-política de reconstrucción de viviendas rurales de ambos procesos. Se utilizó una metodología cualitativa, realizando 15 entrevistas y un focus group con actores claves, además de analizar información secundaria para caracterizar los impactos y los instrumentos de política habitacional aplicados. Los resultados dan cuenta de la diversidad de enfoques utilizados
COMPARATIVE ANALYSIS OF THE TECHNICALPOLITICAL LOGIC OF RECONSTRUCTION: EARTHOUAKE AND FOREST FIRES IN MAULE, CHILE

\section{Abstract}

The forest fires of 2017 caused significant damage in the Maule Region, and forced a new process of reconstruction in rural areas that had been affected by the 2010 earthquake. This paper aims to identify the main differences in the technical-political logic of reconstruction of houses of each process. Using a qualitative methodology 15 interviews and a focus group with key actors were undertaken, along with analysis of secondary information to characterize the impact and identify the housing policy tools applied. The evidence shows the diversity of the approaches used in reconstruction. On the one hand, important advances were detected, such as 
para reconstruir. Por un lado, se advierten importantes avances, como la adecuación de las normativas técnicas y los instrumentos (subsidios), una mayor apertura a los procesos participativos, una planificación realizada por el Estado, y un control del voluntarismo de grupos privados. Por otro lado, se evidencia la existencia de desafíos pendientes en el ámbito de la estructura institucional, las relaciones entre actores relevantes y la participación comunitaria. Con todo, en el proceso de reconstrucción posterior a los incendios forestales, se aprecia un Estado que asume un mayor rol en la coordinación de los actores involucrados y de acompañamiento de las comunidades.

\section{PALABRAS CLAVE: RECONSTRUCCIÓN; INCENDIOS FORESTALES; TERREMOTO; VIVIENDA; RURALIDAD.}

Recibido: 2019-06-11

Aceptado: 2019-12-12

1 Proyecto "Sistematización del proceso de reconstrucción post incendios forestales en la Región del Maule", financiado por el MIDESO; Proyecto MEC 80170032, financiado por CONICYT

2 Chile. Académico de la Escuela de Sociología e investigador del Centro de Estudios Urbano-Territoriales (CEUT) de la Universidad Católica del Maule, http://orcid.org/00000001-8809-5486. Correo electrónico: smicheletti@ucm.cl the adaptation of technical regulations and policy (subsidies), greater openness to participatory processes, and planning carried out by the State and not left to the voluntarism of private groups. On the other hand, it was also possible to detect the existence of pending issues in the institutional structure of reconstruction, relations between relevant actors and community participation. Overall, in the process of reconstruction following forest fires, the State has assumed a greater role in the coordination of those actors involved and of support of the affected communities.

\section{KEYWORDS: RECONSTRUCTION; FOREST WILDFIRES; EARTHOUAKE; HOUSING; RURALITY.}

Received: 2019-06-11

Accepted: 2019-12-12

3 Chile. Investigador independiente, http://orcid.org/00000002-8918-2781. Correo electrónico: dantepancani@hotmail.com

4 Italia. Académica e investigadora del Dipartimento Territorio e Sistemi Agro-Forestali, Universitá degli Studi di Padova, http://orcid.org/0000-0002-8918-2781. Correo electrónico: elena.pisani@unipd.it 


\section{Introducción}

Los incendios forestales que entre el 18 de enero y el 5 de febrero de 2017 recorrieron el territorio chileno -desde la Región de Coquimbo hasta la Araucanía- afectaron un total de 467.537 hectáreas (Corporación Nacional Forestal [CONAF], 2017a). De los 681 focos detectados en esas dos semanas, 471 se concentraron en las regiones de O'Higgins, Maule y Biobío, que resultaron ser las más golpeadas. El saldo del desastre incluyó a miles de damnificadas/os e ingentes pérdidas materiales. Para la Región del Maule significó el comienzo de un nuevo proceso de reconstrucción, luego de haber sido golpeada severamente por el terremoto del 27 de febrero del 2010. En ambos casos, los sectores rurales fueron ampliamente afectados. En este contexto, el trabajo realiza un análisis comparativo de lógica técnico-política de reconstrucción de viviendas rurales de ambos procesos, tratando de generar un aporte para el mundo público en la explicitación de las lógicas de intervención estatal, la fijación de estándares y la identificación de temas pendientes y desafíos para futuros eventos.

\section{Revisión de literatura}

La gobernanza de la gestión del riesgo de desastre es un tema que a nivel internacional se ha tratado y normado a través de distintos instrumentos; los principales son el Marco de Acción de Hyogo y el posterior Marco de Sendai para la Reducción del Riesgo de Desastres 2015-2030. En este último documento, se establece como prioridad el fortalecimiento de la gobernanza, vinculada esencialmente a la generación de objetivos, planes, competencia, directrices y coordinación en los sectores y entre ellos, así como a la participación de los actores pertinentes (ONU, 2015, p. 17).

En Chile, el Estado ha asumido, históricamente, posiciones diversas frente a los procesos de reconstrucción posteriores a las catástrofes, dependiendo del contexto socio-político. En ese sentido, Letelier y Boyco (2011) identifican al terremoto de Valparaíso de 1985 como un hito que marca un cambio de enfoque en relación al rol del Estado; hasta ese momento los desastres fueron oportunidades para los territorios y sus ciudadanos, y se innovó a nivel normativo, legal y de estructura político-administrativa. Desde los años ochenta en adelante -de la mano con la instalación de un modelo socio-económico neoliberal- el Estado se transformó en un actor principalmente subsidiario.

La reconstrucción posterior al sismo del 2010 -último evento catastrófico de magnitud nacional- ha sido objeto de numerosos análisis. A nivel internacional, resumimos dos visiones; por un lado, investigadores como Comerio (2015) destacaron el proceso de reconstrucción caracterizándolo por la presencia de un fuerte liderazgo a nivel nacional y local, por la adaptación de programas habitacionales para satisfacer 
las necesidades de las condiciones locales, la combinación de la gestión del gobierno nacional con la contribución de los ciudadanos locales, la planificación que creó una oportunidad para expandir la política urbana nacional y el desarrollo de un marco para la participación ciudadana a nivel local. Una visión diversa representa el trabajo de un equipo internacional encabezado por Irazábal (2015), que arriba a resultados opuestos, señalando graves carencias institucionales, un marcado sectorialismo y la falta de coordinación en la gestión de la reconstrucción, además de la ausencia de un Plan de Acción a nivel nacional.

En Chile, el análisis académico se acercó mucho más a esta segunda perspectiva. Un importante número de publicaciones abordó el tema desde la perspectiva urbana, centrándose en el efecto del desplazamiento de damnificados de bajos ingresos desde los sectores centrales de las ciudades intermedias hacia la periferia (Cárdenas y Fuster, 2017; Letelier y Boyco, 2011; Letelier y Rasse, 2016). En esta línea, se abordaron también los efectos socio-espaciales de la lógica de reconstrucción, destacando los procesos de desplazamiento subsidiario y gentrificación (Inzulza y Cárdenas, 2017; Inzulza, Maragaño, Boano y Díaz, 2018), así como la importancia de la escala barrial y su cohesión social para la recuperación de la ciudad intermedia maulina (Inzulza, 2014).

Desde la perspectiva rural, diversos trabajos pusieron en evidencia que las políticas de reconstrucción no reconocieron las particularidades del territorio, provocando el incremento de la precariedad en el habitar, la pérdida de patrimonio e identidad, y la profundización de los fenómenos de desplazamiento de la población hacia la periferia de las ciudades (Concha, Micheletti, Olivares y Rasse, 2015; Concha y Rasse, 2014; Micheletti y Letelier, 2016; Micheletti, Opazo, Rodríguez, Spuler y Ríos, 2017); conclusiones que fueron corroboradas a cuatro años de ocurrido el terremoto, por el "Diagnóstico Estado de la Reconstrucción - Terremoto y Tsunami 27 de Febrero de 2010" y el posterior "Plan de Cierre Reconstrucción Terremoto y Tsunami 27F, 2010" de la Delegación Presidencial para la Reconstrucción (2014 y 2015).

El ámbito de la planificación del proceso de reconstrucción tuvo especial relevancia en las investigaciones, que, por un lado, cuestionaron la lógica del Estado chileno de delegar su responsabilidad a entes privados -a través de asociaciones formuladas en marcos institucionales débiles, donde la articulación entre los actores no es clara- replicando, además, las deficiencias de la planificación urbana y regional en Chile: integralidad baja y participación ciudadana deficiente (González, 2017; Herrmann, 2015; Imilan y González, 2017). Por otro, se generaron algunas propuestas para mejorar la capacidad de los instrumentos de planificación de resolver la necesidad de espacios públicos/comunes/intersticiales para despertar el carácter participativo de los usuarios (Intveen, 2015; Prieto, 2018). 


\section{Problemática y estado del arte: incendios y reconstrucción en la Región del Maule}

El Maule es un territorio emplazado al sur de la Región Metropolitana, y habitado por poco más de un millón de habitantes; está conformado por cuatro provincias (Talca, Curicó, Linares y Cauquenes), siendo la ciudad de Talca el centro poblado más importante y la capital regional. Es un territorio altamente productivo y exportador, especialmente en los ámbitos agropecuario y forestal (Cabello, 2015).

Analizando transversalmente la región, se pueden distinguir claramente tres franjas: la primera corresponde al sector de cordillera y pre-cordillera; luego se reconoce el valle central -caracterizado por la presencia de suelos fértiles y la existencia de un sistema agroindustrial altamente desarrollado; y, finalmente, el sector del secano (secano interior y secano costero), que cuenta con terrenos de muy baja productividad agrícola y que en las últimas décadas se ha poblado con grandes extensiones de plantaciones forestales exógenas (esencialmente Pinus radiata y Eucalyptus).
Es justamente en esta última área donde se produjeron los incendios del verano 2017, que a nivel regional afectaron a 279.931 ha: 185.875 ha de plantaciones forestales (66,4\%), 29.588 ha de bosque nativo (10,6\%), 51.055 ha de praderas y matorrales (18,2\%), 12.551 ha de terrenos agrícolas $(4,5 \%)$ y 862 ha de otros usos de suelo como áreas urbanas, humedales y otras áreas sin vegetación (0,3\%) (CONAF, 2017b).

En relación a las pérdidas materiales en el ámbito agrícola, resultaron damnificados 2.117 agricultores, y se perdieron 5.319 cabezas de ganado bovino, 3.523 de ganado equino, 22.439 de ganado ovino y caprino, y 26.183 colmenas (Barrera, 2017).

El análisis de la $\mathrm{FIBE}^{5}$ - Ficha Básica de Emergencia del Ministerio de Desarrollo Social permitió identificar a 8.360 personas damnificadas (6.734 en la Provincia de Talca, 1.240 en la Provincia de Linares, 785 en la Provincia de Curicó y 427 en la Provincia de Cauquenes). La comuna más golpeada fue, sin duda, Constitución -con 4.681 damnificados- donde se emplazaba la localidad rural de Santa Olga, único centro poblado que fue arrasado completamente por el siniestro (aprox. 850 viviendas). Del total de personas afectadas, 4.458 registraron daños totales en su vivienda, 252 declararon que la vivienda resultó muy afectada, 271

5 La FIBE considera una "declaración” de daño en vivienda. 
medianamente afectada y 386 poco afectada. En términos generales, este número equivale a aproximadamente 3.068 familias $^{6}$

En relación a la ayuda otorgada por el Ministerio de Vivienda y Urbanismo [MINVU], el Plan de Reconstrucción contempla 1.209 soluciones habitacionales para la Región del Maule, considerando viviendas a reparar, reposición de la vivienda en el mismo terreno, relocalización mediante subsidios de adquisición o la incorporación a proyectos en desarrollo, y la construcción de un conjunto habitacional en la localidad Santa Olga de Constitución.

Más en detalle, el MINVU ${ }^{7}$ señala que se utilizaron estos instrumentos (subsidios):

- Subsidio Banco de Materiales. Corresponde a la entrega de un subsidio destinado a la reparación de viviendas cuyo daño es leve y/o moderado. Permite la adquisición de materiales de construcción a través de proveedores locales que deben suscribir un convenio.

- Subsidio de Construcción en Sitio Residente. Destinado a la construcción de una vivienda definitiva, para damnificados que disponen de terreno emplazado en zona segura.

6 Calculado en base al número de personas indicadas como jefes de hogar en la Ficha Básica de Emergencia

7 Reporte plan de reconstrucción incendio forestal Maule. Información obtenida vía Ley de Transparencia. Código solicitud: CAS-5635088-03Y9G2
- Subsidio para la Construcción de un Nuevo Conjunto Habitacional. Es un instrumento colectivo, destinado a generar oferta habitacional para damnificados de los sectores de Santa Olga, Los Aromos y Altos de Morán, cuyas viviendas estaban emplazadas en zonas de riesgo, irregulares y/o dentro de otros predios.

- Subsidio para la Adquisición de Viviendas. Destinado a la compra de viviendas nuevas o usadas, destinado a quienes no disponen de terreno seguro, o bien a grupos familiares allegados o arrendatarios, que optan voluntariamente por relocalizarse.

Cabe destacar que -a nivel operativo- se utilizaron dos grandes categorías para la intervención estatal: lo que se denominó como "reconstrucción urbana"8 (Santa Olga, Altos de Morán y Los Aromos), que considera obras y equipamiento urbano y lo que denominó "reconstrucción de la ruralidad dispersa" (el resto de los casos, repartidos en diferentes localidades rurales de la Región). Como se puede apreciar en la Tabla 1 , respecto del avance en la entrega de subsidios, construcción y/o compra de viviendas, el MINVU informa que, al 31 de marzo del 2019, existen 1.199 soluciones habitacionales vigentes (que corresponde al 99,9\% del universo de

En términos conceptuales diferimos de esta definición, pero entendemos que se vinculó al proceso de "urbanización" del sector. 
subsidios por entregar), con diversos estados de avance.

Adicionalmente, existía un número importante de damnificados que no podían acceder a subsidios por poseer otras propiedades habitacionales ${ }^{9}$ y/o que voluntariamente no participaron de la reconstrucción con financiamiento estatal, y fueron atendidos mediante el aporte de donaciones privadas y de las campañas públicas realizadas.

\section{Metodología}

Buena parte de los trabajos mencionados en la revisión de literatura, adoptaron un enfoque metodológico de tipo cualitativo, para abordar de manera profunda los tópicos de análisis vinculados a las subjetividades de quienes sufrieron la catástrofe y vivieron el posterior proceso de recuperación. Es una perspectiva que -junto a la consulta de fuentes secundarias cuantitativas para la caracterización del impacto en la infraestructura y la población- adoptamos para nuestro trabajo, centrado en descifrar la lógica

9 Criterio establecido por el MINVU para la entrega de subsidios de la reconstrucción posterior a los incendios forestales del 2017, desde la perspectiva estatal.

En términos metodológicos, la primera etapa fue la recopilación de información secundaria relativa a los impactos y los damnificados en la Región del Maule. Para cuantificar los afectados del siniestro, se realizó un análisis de la FIBE Ficha Básica de Emergencia ${ }^{10}$ del Ministerio de Desarrollo Social.

Como se puede apreciar en la Tabla 2, con el objetivo de trabajar los objetivos fijados para la investigación, se realizaron entrevistas semiestructuradas a las 10 principales autoridades (nacionales y locales) y funcionarios públicos vinculados a la reconstrucción (tanto del segundo mandato de la Presidenta Michelle Bachelet, como del segundo mandato del Presidente Sebastián Piñera, que inició el 11 de marzo de 2018). A la vez, se entrevistaron a otros 6 actores relevantes del tercer sector, empresa y academia local.

10 La Ficha Básica de Emergencia (FIBE), “tiene por objetivo levantar información más detallada de los grupos familiares, para dar apoyo en la respuesta de la emergencia. Este instrumento es parte del Sistema de Evaluación de Daños y Necesidades reconocidos por el Estado de Chile para situaciones de emergencias, desastres y catástrofes (DS. 156 del 2002 y D.S. 697 del 2015) y reemplaza la anterior Encuesta Familiar Única (EFU). 
TABLA 1. RESUMEN DE SUBSIDIOS ASIGNADOS Y AVANCES POR MODALIDAD Y SECTOR: SECTOR RURAL DISPERSO (SRD) Y SECTOR SANTA OLGA

\begin{tabular}{|c|c|c|c|c|}
\hline \multirow[t]{2}{*}{ Modalidad } & \multirow[t]{2}{*}{$\begin{array}{c}\text { Soluciones habitacionales } \\
\text { vigentes }\end{array}$} & \multicolumn{3}{|c|}{$\begin{array}{c}\text { Soluciones habitacionales } \\
\text { vigentes }\end{array}$} \\
\hline & & $\begin{array}{l}\text { Soluciones } \\
\text { ejecutadas }\end{array}$ & En ejecución & Sin inicio \\
\hline $\begin{array}{l}\text { SRD - Banco de } \\
\text { Materiales }\end{array}$ & 33 & 31 & 2 & 0 \\
\hline $\begin{array}{l}\text { SRD - Construcción } \\
\text { Sitio Propio }\end{array}$ & 200 & 168 & 27 & 5 \\
\hline SRD - Adquisición & 138 & 97 & 41 & 0 \\
\hline $\begin{array}{l}\text { Subtotal Subsidio } \\
\text { Rural Sectores Dis- } \\
\text { persos (SRD) }\end{array}$ & 371 & 296 & 70 & 5 \\
\hline$\%$ & $100 \%$ & $79,8 \%$ & $18,9 \%$ & $1,3 \%$ \\
\hline $\begin{array}{l}\text { Santa Olga - Banco de } \\
\text { Materiales }\end{array}$ & 3 & 3 & 0 & 0 \\
\hline $\begin{array}{l}\text { Santa Olga - Cons- } \\
\text { trucción Sitio Propio }\end{array}$ & 368 & 88 & 135 & 145 \\
\hline $\begin{array}{l}\text { Santa Olga - Construc- } \\
\text { ción Nuevos Terrenos }\end{array}$ & 270 & 0 & 270 & 0 \\
\hline $\begin{array}{l}\text { Santa Olga } \\
\text { - Adquisición }\end{array}$ & 187 & 134 & 53 & 0 \\
\hline Subtotal Santa Olga & 828 & 225 & 458 & 145 \\
\hline$\%$ & $99,9 \%$ & $27,1 \%$ & $55,2 \%$ & $17,5 \%$ \\
\hline TOTAL & 1.199 & 521 & 528 & 150 \\
\hline$\%$ TOTAL & $99,9 \%$ & $43,4 \%$ & $44,0 \%$ & $12,5 \%$ \\
\hline
\end{tabular}

Fuente: MINVU, 2019 (Información obtenida vía Ley de Transparencia. Código solicitud: CAS-5635088-Q3Y9G2) 


\begin{tabular}{|c|c|}
\hline Tipología & Actor \\
\hline Autoridad nacional & Coordinador Nacional para la Reconstrucción (Gobierno Bachelet) \\
\hline Autoridad nacional & Ministra de Vivienda y Urbanismo (Gobierno Bachelet) \\
\hline Autoridad nacional & Jefe de la Comisión Asesora de Reconstrucción del MINVU (Gobierno Bachelet) \\
\hline Autoridad nacional & Jefa de Comisión Asesora de Reconstrucción del MINVU (Gobierno Piñera) \\
\hline Autoridad local & Intendente de la Región del Maule (Gobierno Bachelet) \\
\hline Autoridad local & Alcalde Municipalidad de Constitución (Gobierno Bachelet y Piñera) \\
\hline Funcionario público & SEREMI Maule de Vivienda y Urbanismo (Gobierno Bachelet) \\
\hline Funcionario público & SEREMI Maule de Vivienda y Urbanismo (Gobierno Piñera) \\
\hline Funcionario público & Encargado de reconstrucción MINVU Maule (Gobierno Bachelet) \\
\hline Funcionario público & $\begin{array}{l}\text { Encargada FIBE SEREMI Maule Ministerio Desarrollo Social y Familia (Gobierno } \\
\text { Bachelet y Piñera) }\end{array}$ \\
\hline Tercer sector & Director Regional de la Fundación para la Superación de la Pobreza Maule \\
\hline Tercer sector & Coordinadora provincial de Talca Desafío Levantemos Chile \\
\hline Academia & Profesional Observatorio Laboral FACSE UCM \\
\hline Academia & $\begin{array}{l}\text { Docente escuela de Psicología UCM - Proyecto de monitores salud mental es- } \\
\text { cuela de Santa Olga }\end{array}$ \\
\hline Academia & $\begin{array}{l}\text { Docente escuela de Psicología UCM - Proyecto de monitores salud mental es- } \\
\text { cuela de Santa Olga }\end{array}$ \\
\hline Empresa & Encargado de Red de prevención comunitaria ACERCAREDES. \\
\hline
\end{tabular}

Fuente: elaboración propia 
Las entrevistas se transcribieron y sistematizaron en una matriz, para ser estudiadas utilizando el análisis de contenido (Bardin, 1996; Krippendorff, 1997; Piñuel, 2002), según categorías pre-establecidas y otras que emergieron después, a saber: principios orientadores, enfoque de gestión, coordinación institucional, planificación estatal y participación de actores sociales y económicos.

Finalmente, se realizó un focus group con 18 asistentes, donde estuvieron presentes ex autoridades nacionales y locales, dirigentes sociales de comunidades afectadas, académicos y representantes de la empresa forestal Arauco, con el objetivo de generar un espacio de diálogo y reflexión centrado en el rol del Estado a partir del caso de los incendios forestales en el Maule. Específicamente, se abordaron dos temas centrales: la relación entre la planificación / gestión territorial y la ocurrencia de desastres de origen antrópico, como los incendios, y el carácter de la reconstrucción del hábitat (vivienda y espacios públicos). También el focus se transcribió y sistematizó para ser analizado (Onwuegbuzie, Dickinson, Leech y Zoran, 2011).

\section{Resultados: una aproximación comparativa a la lógica técnico-política de reconstrucción en el Maule}

\section{LA RECONSTRUCCIÓN RURAL POST TERREMOTO}

El sismo de 8,8 grados Richter, que sacudió al país en febrero de 2010, dejó un saldo de 76.581 de soluciones de viviendas a desarrollar (23.879 a reponer y 52.702 a reparar) en la Región del Maule, lo que correspondió al 21\% del total de viviendas dañadas del país. Según estimaciones oficiales, en el Maule se contabilizaron alrededor de 65.000 familias damnificadas, y 12 de las 28 comunas críticas a nivel nacional estaban en el Maule (Letelier y Boyco, 2011). En el contexto rural, además de las viviendas y la infraestructura productiva, caminos, puentes, sistemas de agua potable rural y caletas de pescadores -entre otros- sufrieron fuertes daños. Cabe destacar que, finalmente, no existieron datos oficiales de población rural afectada y tampoco registro de los daños y pérdidas de la ruralidad más profunda (Delegación Presidencial para la Reconstrucción, 2014). 
Como ya mencionamos, el proceso de reconstrucción consiguiente ha sido objeto de varios análisis a nivel nacional; los autores identificaron de manera bastante consensuada un conjunto de críticas vinculadas a la acción del Estado, que tratamos de sintetizar a través de estos puntos:

- Los programas de reconstrucción operaron desde una lógica neoliberal (Imilan y González, 2017), orientada a la reposición de viviendas a través del mercado inmobiliario y basada en una actuación del Estado que se puede resumir de esta manera: subsidio a la demanda, incentivo a la participación del sector privado y organizaciones intermedias, bajo control del proceso, escaso acompañamiento a los damnificados, rol de intermediador entre damnificados y empresas, generación de valor social en la lógica de oferta y demanda (Concha et al., 2015; Micheletti y Letelier, 2016).

- No se constituyó -en el contexto de una catástrofe enorme, que marcó un antes y un después a nivel nacional- una institucionalidad específica, ad hoc, para liderar el proceso y trabajar de manera intersectorial. A la vez, se siguieron utilizando -con mínimas modificaciones- los mecanismos y programas regulares de acceso a la vivienda (subsidios), pese a la excepcionalidad de la situación (Letelier y Boyco, 2011). Esta continuidad de las políticas previas se interpretó a la vez como fortaleza y limitación.
Donde sí se registraron retrocesos significativos a políticas propias de los 90', fue en la ampliación del rol del sector privado para definir unilateralmente la oferta (localización y estándar) y en la baja participación efectiva de los damnificados.

- Al no existir instrumentos de políticas adecuados, el escaso interés de las empresas constructoras por reconstruir viviendas en el sector rural -debido esencialmente a los costos asociados a la dispersión y aislamiento- significó un avance significativamente más lento, en comparación con los sectores urbanos; ocurriendo con frecuencia casos de abandono de obras (Micheletti y Letelier, 2016). Se creó, en fin, lo que podríamos denominar una "falla de mercado", siendo una inequidad que los incentivos no lograron corregir.

- La falta de pertinencia social, económica y cultural de las soluciones habitacionales -propuestas por el Estado para el sector rural-, vinculada a la oferta homogénea a nivel nacional y basada en el supuesto que la tipología de vivienda social urbana era de uso universal, sin considerar si fuese apropiada también para las familias rurales (también en consideración de su diferente especialización productiva) (Concha et al., 2015).

- Las políticas de reconstrucción alimentaron procesos de migración desde lo rural hacia la periferia de las ciudades intermedias, debido a la concentración de la oferta de 
viviendas para damnificados en esos sectores (Letelier y Boyco, 2011; Micheletti et al., 2017). Esto se ha expresado a través del uso de la modalidad CNT - Construcción en Nuevos Terrenos- desarrollada, esencialmente, en función del suelo disponible, en su mayoría de propiedad o gestionado por las empresas constructoras. En el Maule, fueron 134 los proyectos habitacionales de este tipo, y 13.524 las familias atendidas por esta modalidad (Delegación Presidencial para la Reconstrucción, 2015). Además de las complicaciones vinculadas a la nueva localización y calidad de las obras, los mayores problemas que presentaron estos conjuntos habitacionales, una vez que fueron habitados por las familias, fueron a) la relación entre vecinos; b) defectos en la calidad de la construcción, proyectos incompletos y necesidades de mantención y c) las áreas comunes (Delegación Presidencial para la Reconstrucción, 2015).

- Finalmente, se criticó con mucha fuerza la planificación del proceso, ya que los Planes de Reconstrucción Urbana y de Reconstrucción del Borde Costero tuvieron un impacto limitado por la debilidad de los lazos entre el sector público y el sector privado, la falta de un enfoque amplio y la falta de involucramiento de los actores locales (Imilan y González, 2017). También cabe señalar que "los actores privados no desarrollaron intereses específicos en las localidades afectadas como oportunidades de negocios, por lo tanto, no pasaron la etapa de ser una simple declaración de intenciones" (González, 2017, p. 115).

Evidentemente, esta conculcación de derechos provocó un aumento de la vulnerabilidad del hábitat rural que se expresó con fuerza a través de la pérdida de identidad y patrimonio y aceleración del proceso de migración hacia la ciudad.

\section{LA LÓGICA DE RECONSTRUCCIÓN RURAL LUEGO DE LOS INCENDIOS FORESTALES}

Posterior al 27F el Estado de Chile ha tenido que intervenir en desastres socio-naturales acontecidos en otras regiones: el terremoto en el Norte Grande y el incendio de Valparaíso (2014); las inundaciones en el Norte, la erupción del Volcán Calbuco, el aluvión de Tocopilla y el terremoto y tsunami de Coquimbo (2015); el terremoto de Quellón (2016); el incendio de Playa Ancha y las inundaciones en el Norte Chico (2017) y el aluvión en la Villa Santa Lucía (2018). Estas experiencias han ido consolidando una serie de conocimientos técnico-políticos -vinculados a la reconstrucción de viviendas- que queremos tratar de reconocer en relación a los incendios forestales del verano 2017 (último proceso de reconstrucción del periodo 2014-2018). En este sentido, las entrevistas realizadas durante el estudio nos permiten distinguir algunos rasgos principales de la experiencia. 


\section{LOS PRINCIPIOS ORIENTADORES DE LA RECONSTRUCCIÓN}

El MINVU señaló que la reconstrucción posterior a los incendios forestales se inspiró en el principio general de "Reconstruir Mejor", ampliamente difundido en la Conferencia de Naciones Unidas para la Reducción del Riesgo de Desastres -celebrada en Sendai, Japón en 2015. Para ello, señaló haberse ajustado a principios rectores para tomar decisiones y acciones, tanto para la labor en vivienda, como en infraestructura y ordenamiento territorial: seguridad, calidad, intersectorialidad y participación. Confrontar el resultado con estos principios, muestra un énfasis distinto respecto de procesos anteriores, que se orientaron a la rapidez como criterio de resultado. El primero de ellos (seguridad), define que la reconstrucción debe reconocer los riesgos y mitigarlos, conectando con la manera en que se usa el territorio. Estrechamente ligada al segundo principio (calidad), se explicitó la necesidad de considerar procesos y estándares técnicos para asegurar el correcto resultado de las soluciones habitacionales.

"Nos vamos a demorar, pero vamos a vivir mejor, y la gente lo va a valorar mejor, uno entiende la impaciencia, uno entiende que la gente quiere un lugar, una casa, un techo, pero yo quiero una casa, un techo, agua, alcantarillado, vereda, luz, calles, (...) entonces para lograr eso tengo que también ser bastante paciente. (...) Si nos hubiésemos limitados todos a hacer casas y solo a devolver una vivienda y decirle a la gente 'ahí está lo que ustedes tenían', hubiésemos vulnerado los derechos de la gente" (Autoridad local)

El tercer principio declarado (intersectorialidad) da cuenta de la necesidad de articulación para actuar sobre el territorio, lo que resulta evidente en el caso de Santa Olga; ello supone una visión conjunta del desarrollo local. El cuarto principio (participación), comprende tanto la pertinencia (vínculo entre la reconstrucción, la naturaleza de los territorios y las comunidades, así como las oportunidades y los recursos que brinda el entorno local), como la importancia del involucramiento de la comunidad en el proceso; por ejemplo: en labores de catastro, diagnóstico, planificación e implementación de la reconstrucción.

\section{EL ENFOQUE DE GESTIÓN DE LA RECONSTRUCCIÓN}

El enfoque de gestión usado, que devela la lógica de acción estatal, se centró en la asignación de subsidios en base a los programas vigentes; sin embargo, se introdujo un conjunto de modificaciones tendientes a facilitar el acceso y su operación, recurriendo a la facultad que los mismos decretos otorgan, para realizar llamados en condiciones especiales en caso de desastres socio-naturales. Las principales modificaciones respecto del acceso fueron: eximir de la acreditación de un instrumento de caracterización socioeconómica, introduciendo únicamente la limitación de tener otras propiedades habitacionales 
(flexibiliza la focalización, se asimila a una política de acceso universal); eximir del requisito de acreditar ahorro previo; multiplicar las alternativas y tiempos para acreditar disponibilidad de terreno (no únicamente en propiedad).

Las principales modificaciones respecto de la aplicación de subsidios (que tienen impacto en la calidad de la solución de vivienda) fueron: la flexibilización del cuadro normativo de recintos y superficies (eximiendo el requisito del tercer dormitorio, sin disminuir superficie total construida); en el esquema de financiamiento de la vivienda, la consideración de recursos especiales para la condición de ruralidad -como habilitación de suelo, soluciones sanitarias especiales, accesibilidad universal y la consideración de subsidios adicionales para introducir sistemas de calefacción, implicando un incremento global del subsidio base.

"Las viviendas del mundo rural son viviendas que tienen entre los 57, 58,60, hasta 75 metros cuadrados construidos, terminados $100 \%$ (...). Nosotros dijimos siempre que (...) las viviendas se construían bajo la lógica del DS $\mathrm{N}^{\circ} 01$, esto es construcción de viviendas en zonas urbanas, adaptado en este caso al mundo rural, que tuviera a lo menos $800 \mathrm{UF}^{11}$ (...) y que esas viviendas iban a ser pagadas al contratista que construyera si, y solo si, tuviesen recepción municipal. (Funcionario público)

Se promovió el uso de la modalidad de "construcción de vivienda y pago posterior" que otorga mayor libertad a las empresas para construir y acreditar el cierre del proceso con la recepción final de obras que otorga la municipalidad. En el caso de la reparación de viviendas, los subsidios consideraron recursos para adecuarlas a estándares vigentes en materia de seguridad. Pero, especialmente, se dispuso de la modalidad de reparación a través de la entrega de una Tarjeta Banco de Materiales (bono en dinero), que permitía la compra de materiales de construcción en instituciones locales en convenio. Los convenios privilegiaron a pequeños proveedores locales por sobre las cadenas, y se buscó reconocer el aporte de los damnificados en gestionar directamente su solución habitacional, aportando la mano de obra, pero también generando impacto en la economía local.

\section{Coordinación institucional}

En el ámbito de la institucionalidad, nos parece importante destacar que, producto de los incendios forestales en las Regiones de O'Higgins, Maule y Biobío, el proceso contó con un Coordinador Nacional de Reconstrucción, cargo similar a los Delegados Presidenciales de 2014 por el incendio en Valparaíso y Terremoto del

11 La unidad de fomento (UF) es una unidad de cuenta usada en Chile, reajustable de acuerdo con la inflación 
Norte Grande. Esta figura fue muy relevante, ya que aseguró la coordinación entre la institucionalidad pública, privada y comunitaria y operó como enlace entre la realidad regional y los equipos nacionales. El Coordinador operó desde la capital -Santiago-, manteniendo interlocución directa con sus pares ministeriales y asegurando la coordinación central, estando, además, localizado periódicamente (de forma semanal) en la región, ayudando en la interlocución entre el nivel nacional y local. Por otro lado, para abordar los casos existentes en sectores rurales dispersos, se nombró una coordinación regional específica.

Como ya se mencionó, llamó la atención por generar una respuesta pertinente, contextualizada, que trató de ponerse en relación con los recursos locales; esto se expresó en varios ámbitos. A nivel institucional, hubo una alta participación de la estructura descentralizada del MINVU en la toma de decisiones, y se trabajó con equipos regionales desde el momento del levantamiento de la información. En este ámbito, cabe destacar que, si bien se reconoce una mayor articulación interministerial y entre autoridades, es a nivel de los mandos medios y de los funcionarios en general donde no operó un mecanismo de coordinación permanente.

"Las instancias de coordinación se dan entre autoridades, no entre los funcionarios y eso no sirve de nada, porque la que estaba a cargo era yo y yo no tuve idea de qué hablaba mi Seremi con el Intendente, ni idea" (Funcionario público)

\section{Planificación estatal}

En el ámbito de la planificación, aparece con fuerza el caso de Santa Olga, en referencia al levantamiento de un Plan Urbano Integral de Reconstrucción (PUIR en adelante, socializado como Plan Maestro de Reconstrucción), que promovió una "organización multisectorial de arriba hacia abajo, en la que había un jefe que coordinaba a todas las demás entidades involucradas" (Garcés, 2018, p. 63). A diferencia de los PRES post terremoto -que definieron grandes proyectos urbanos detonantes y aportaron un conjunto de orientaciones para el modo en que debía ser gestionada la recuperación de edificios públicos (Letelier y Boyco, 2011)- el PUIR apuntó a la reposición de los servicios básicos, pero también a la construcción, reposición y mejoramiento de la infraestructura "urbana" y barrial, de los espacios públicos y de la infraestructura para reducir riesgos. De este modo, el desastre adquirió una dimensión colectiva que el post terremoto no tuvo. Por otro lado, la responsabilidad de la planificación fue asumida directamente por el Estado a través del MINVU y consideró la participación de la Ilustre Municipalidad de Constitución y la comunidad local (MINVU, 2018). 


\section{FOTO 1. RECONSTRUCCIÓN EN SECTORES DE "RURALIDAD DISPERSA".}

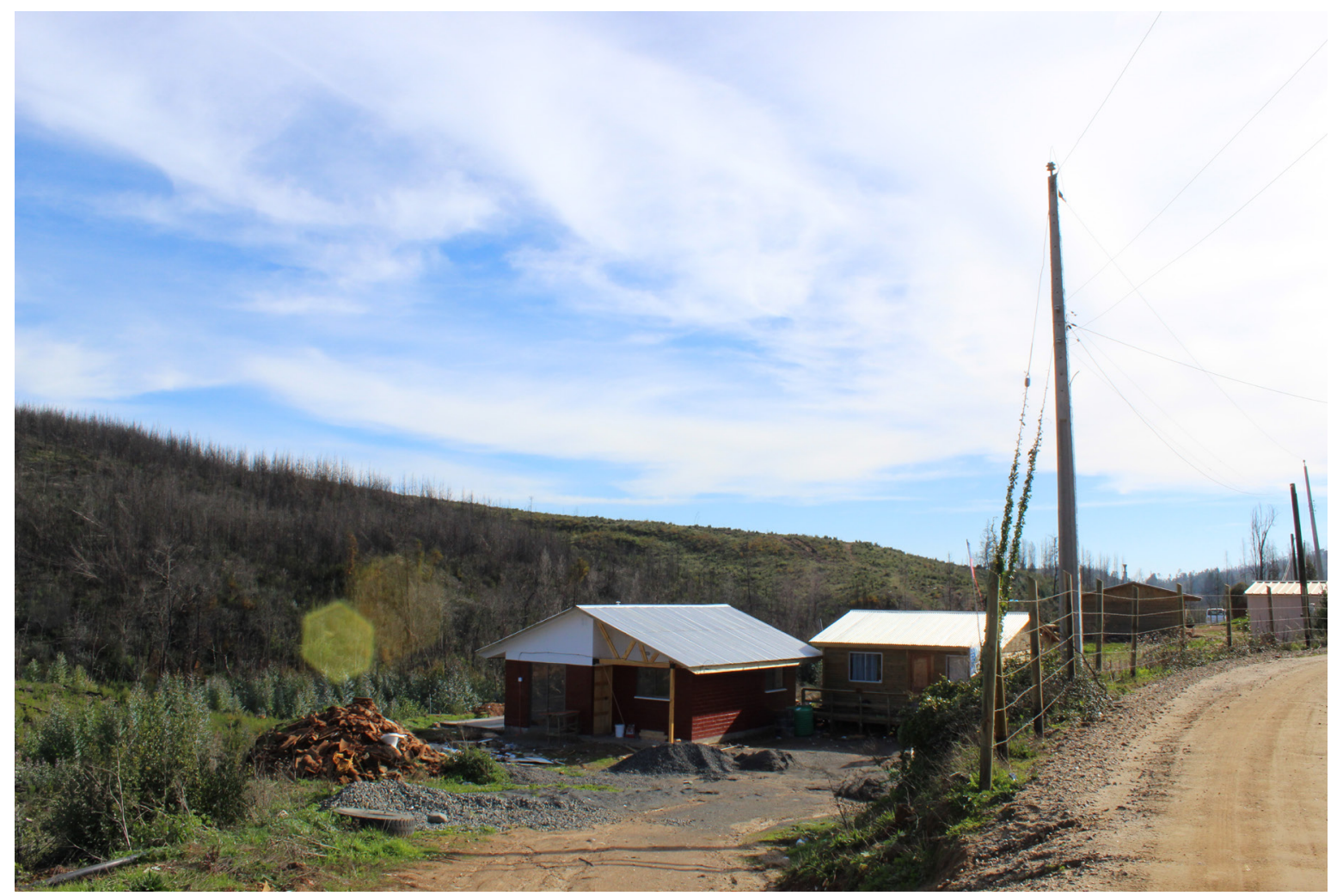

Fuente: autores. 


\section{FOTO 2. RECONSTRUCCIÓN DEL SECTOR DE SANTA OLGA.}

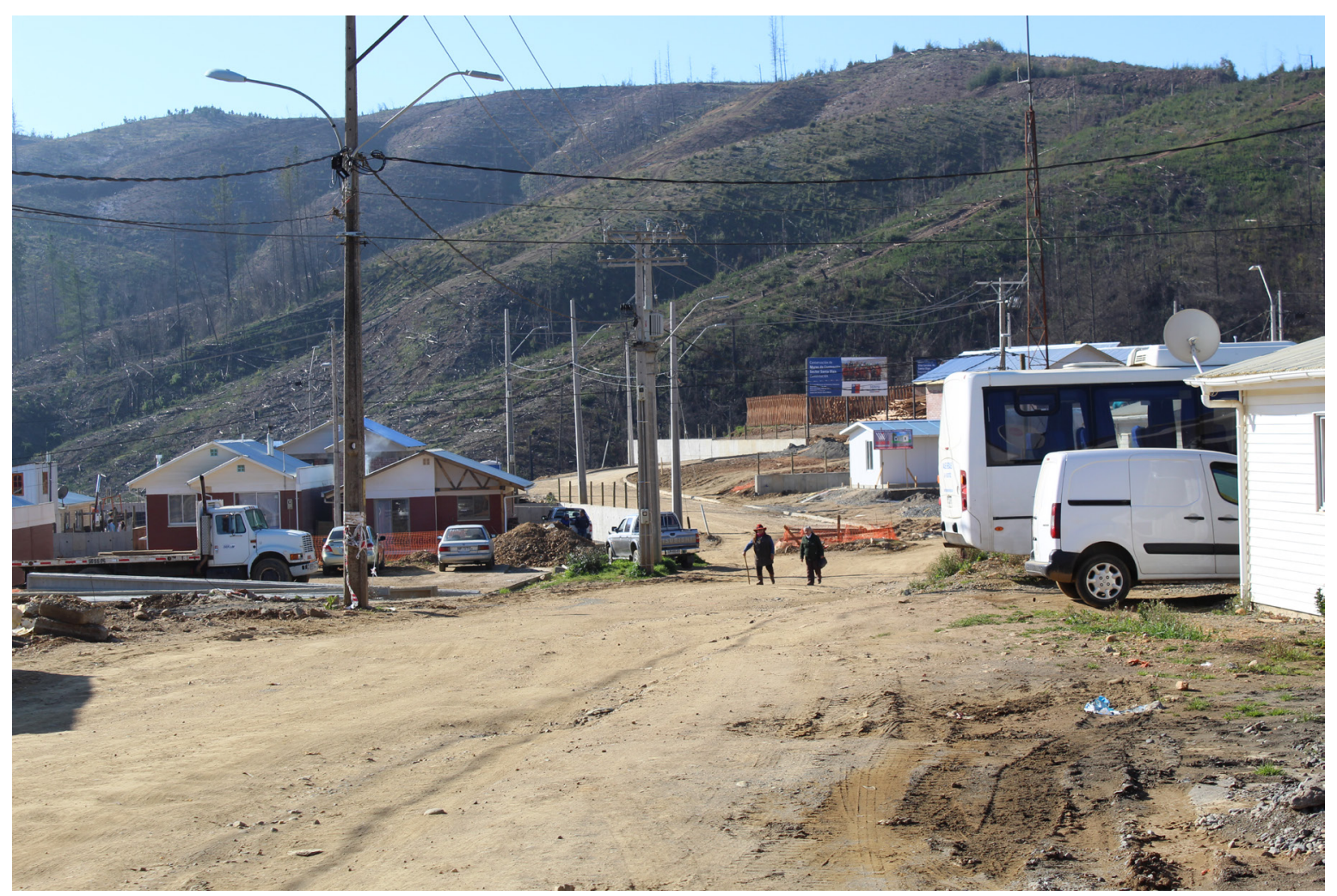

Fuente: autores 


\section{Participación de actores sociales y económicos}

El proceso se destaca por tener una orientación a la economía local: hubo un conjunto de acciones que se ampararon en decisiones políticas y de gestión, que le otorgaron mayor capacidad de conducción y control al ente público, e introdujeron mejores condiciones económicas para actores locales, que no dependían de modificaciones reglamentarias. Fueron convocadas todas las empresas vinculadas a la construcción de viviendas sociales en los sectores afectados y aquellas que decidieran sumarse a la reconstrucción; esto permitió tener un contingente de pequeñas empresas, con arraigo territorial. Se solicitó a las empresas que comprometieran proyectos tipo, de manera que los damnificados pudieran elegir entre alternativas y se promovió el uso diverso de materialidad, en especial considerando la madera. La vinculación entre empresas y damnificados contó con el acompañamiento del Servicio de Vivienda y Urbanización, lo que permitió fijar plazos y actuar en caso de incumplimiento. Para ejercer mayor control y conducción, se fortaleció la dotación de funcionarios, la disponibilidad de recursos y la definición de responsabilidades.

"Desde maestros y empresas locales, uno dice empresas, pero la verdad es que son empresas donde son tres, cuatro maestros donde probablemente el papá con los hijos y que llevan, respetando que el sector tenía preexistente (...) una dinámica económica local, gente que le compra al proveedor local, a la ferretería del barrio, gente que se provee del socio que es el experto que hace los pozos de agua, del otro que es el gasfíter. El sistema lo adecuamos para que lo operaran pequeñas unidades económicas" (Autoridad nacional)

Siempre en el ámbito de las relaciones entre actores, ante la necesidad de atender a todos los damnificados y llegar a los diferentes territorios rurales, se incorporó a nuevas entidades patrocinantes (empresas constructoras, proveedores locales) y se fortaleció el vínculo con las Municipalidades a través de los Encargados Municipales de Emergencia y la Direcciones de Obras Municipales. En este contexto, uno de los aspectos que más destacó fue la difícil convivencia del Estado con algunas organizaciones del tercer sector (fundaciones, ONG, etc.) y los privados (empresas, consorcios económicos). Si bien se valora el aporte que estos entes realizan, hay un contraste de lógicas que subyace en la relación y la complejiza.

"Hay que decirlo bien claro, siempre chocábamos, porque lamentablemente (la organización no gubernamental X) funciona en una lógica distinta, distinta digo en el sentido que ellos buscaban como poder demostrar que el sector privado era siempre más rápido que el sector público" (Funcionario público) 
Si bien los entrevistados reportan un esfuerzo importante en la generación de relaciones positivas con todos los actores, y la definición transversal de estándares mínimos -tanto en la faena constructiva como en los procedimientos administrativos- se generaron algunos conflictos y otras situaciones complejas para las familias damnificadas.

"Todas las viviendas que (la organización no gubernamental X) levantó en el sector rural, ninguna de ellas cuenta con recepción municipal, el compromiso que hicieron firmar a familias del mundo rural, era un compromiso que (...) tú recepcionas la casa, la firmas, firmas el compromiso de recepción conforme, asumiendo que tú serás el responsable de poder continuar con el trámite de conexión a agua potable, conexión a una solución sanitaria" (Funcionario público)

Se trata de un tema que evidentemente no se logró abordar en toda su complejidad (aunque resultó menos desregulado que en el post terremoto), y que seguirá presentándose en las futuras intervenciones estatales, independiente del carácter del desastre a enfrentar. El objetivo es, en este caso, la construcción de asociatividades efectivas y no competitivas.

Un punto aparte es el de la participación comunitaria, que emerge como elemento relevante del proceso; no solamente como un principio general, sino también en su dimensión técnica: "el valor de la participación social, de fortalecer los liderazgos locales, de mantener las redes preexistentes es un potencial, es un activo que tiene un valor incalculable para la calidad de la solución" (Autoridad nacional). Sin duda este principio redunda -desde una perspectiva territorial- en la necesidad de atender de forma específica a las particularidades de los territorios rurales, que se diferencian de los urbanos a nivel identitario-cultural, económico-productivo, político, ecológico-ambiental.

Sobresalió, en este proceso, el trabajo de construcción de cartografías participativas desarrollado por funcionarios del MINVU Maule, en el contexto del diseño del PUIR, así como el trabajo permanente (en el caso de Santa Olga) con la comunidad local organizada, a través de una mesa de trabajo que funcionó como espacio de encuentro, información y discusión de los avances entre las autoridades y la ciudadanía.

Por ejemplo, la decisión de no construir barrios transitorios en los sectores de Santa Olga, Los Aromos y Altos de Moran (marcada por la compleja experiencia del post terremoto), fue una solicitud expresa de los damnificados para evitar afectar la construcción definitiva y las condiciones de seguridad. La etapa de transición de los damnificados fue entonces acompañada por un conjunto de medidas, como el aporte económico permanente para financiar el arriendo y/o los costos de ser allegado, además de la provisión de una vivienda de emergencia 
en terreno seguro y que no afectara la construcción definitiva ${ }^{12}$.

De todos modos, aunque se constituyeron importantes espacios de participación, sigue siendo un tema relevante lo que se denomina como "bajada de la información". Además de las dificultades propias del aparato estatal, queda en evidencia que el trabajo con dirigentes sociales no asegura que la ciudadanía esté informada y se encuentre involucrada en el proceso: "ese vecino también lo que espera que cada uno de nosotros vaya y le diga uno por uno lo que está ocurriendo, cuestión que es absolutamente impracticable" (Funcionario público).

\section{BREVE DISCUSIÓN ACERCA DE LOS RESULTADOS}

Como pudimos apreciar a través de la literatura analizada y de los resultados expuestos, el Estado en Chile ha organizado las reconstrucciones de sectores rurales luego del terremoto 2010 y de los incendios 2017 bajo distintos énfasis, en términos técnico-políticos. Queremos destacar que no es objetivo de este trabajo comparar dos catástrofes -que no son comparables en término de magnitud, extensión territorial y tipo de daño- sino profundizar en las lógicas estatales de intervención.

Así, la reconstrucción post incendios forestales se puede entender en el marco de un proceso de aprendizaje, que incluye aciertos y lecciones asimiladas del $27 \mathrm{~F}$, pero también errores, inconsistencias y-como no- lecturas políticas distintas sobre los hechos, considerando que se alternaron dos gobiernos de tendencias opuestas en la tarea de la reconstrucción. Las entrevistas realizadas nos permitieron vislumbrar diversos desafíos pendientes o no abordados en el ámbito de la estructura institucional, de las relaciones entre actores relevantes, de la participación comunitaria y de la lógica de intervención estatal. Pero también fue posible detectar avances importantes en relación a la experiencia regional posterior al 27F; como la adecuación de las normativas técnicas y los instrumentos (subsidio) a una situación de emergencia, una mayor apertura a los procesos participativos, una planificación de la reconstrucción realizada por el Estado (no dejada en las manos de grupos privados). Asimismo, fue notorio el establecimiento de una coordinación nacional, que operó tanto en el centro como en la región, representando un elemento dinamizador que

12 Cabe destacar que este tipo de apoyo estuvo vigente también en los incendios de Valparaíso en 2014 y 2017, en el terremoto del Norte Grande de 2014, y en las inundaciones de Atacama de 2015. En el caso de los incendios forestales, los sistemas de control para asignar y mantener los bonos, estuvieron condicionados a la efectiva participación de las familias en obtener una solución habitacional definitiva. 
incidió positivamente en el resultado. Finalmente, el rol de los equipos locales y su capacidad de dirigir y desarrollar desde la región, fue uno de los avances más destacados.

Para no extendernos en la discusión, que se desarrolló también en el apartado anterior, en la Tabla 3 proponemos un esquema sintético de comparación entre los dos procesos analizados, utilizando las categorías propuestas anteriormente.

Finalmente, podemos decir que en el último proceso de reconstrucción se apreció un Estado que -a través del Ministerio de Vivienda y Urbanismo y de una estructura centralizada con fuerte participación regional- actuó de manera diferente a como lo había hecho luego del terremoto del 27F. Un Estado que tomó decisiones, que, aunque en algunos casos fueran muy complejas en términos sociales y políticas (pensamos, por ejemplo, en un tema muy debatido políticamente en este momento, que refiere a la oportunidad de reconstruir el pueblo de la Nueva Santa Olga en la ubicación original, considerando las problemáticas de empleo que afectan a una comunidad que en buena parte dependía económicamente del recurso forestal). Un estado que asumió un rol de coordinación con los otros actores del proceso, acompañando a la comunidad. Un Estado que hizo un esfuerzo por determinar estándares técnicos mínimos, pero también por construir acuerdos sociales con las comunidades afectadas. Lejos de querer celebrar en aquello una actuación estatal excepcional, creemos firmemente que estos puntos debieran asumirse como estándares mínimos en caso de desastres socio-naturales.

\section{Conclusiones}

Actualmente, la sociedad vive una exposición permanente al riesgo y nuevos riesgos, asociados al cambio climático (como la sequía y los eventos hidrometeorológicos), se van tornando cada vez más amenazantes. La frecuencia de las catástrofes ha aumentado notablemente a nivel nacional (en el caso específico de los incendios forestales, según CONAF (2019), se registraron 611 eventos en el período 2008-2018, mientras que en la década anterior fueron solamente 371), y es entonces fundamental para el Estado, las empresas y las comunidades seguir aprendiendo de las experiencias enfrentadas, para poder mejorar la eficacia y eficiencia de sus acciones y así reducir los posibles impactos negativos a nivel local. Por otro lado, aumenta también la exigencia de una respuesta oportuna por parte de todos los actores involucrados, especialmente de las comunidades afectadas.

Esperamos con nuestro trabajo poder aportar a la "fijación" de estos pisos mínimos, al explicitar los aprendizajes del proceso y también al facilitar el reconocimiento de los errores como una oportunidad de mejora. Entendemos que esto, además de redundar en un beneficio para las comunidades afectadas, irá mejorando también la percepción, señalada por algunos 
TABLA 3. ANÁLISIS COMPARATIVO DE LÓGICA TÉCNICO-POLÍTICA DE RECONSTRUCCIÓN DE VIVIENDAS RURALES POST TERREMOTO Y POST INCENDIOS FORESTALES EN LA REGIÓN DEL MAULE

\begin{tabular}{|c|c|c|}
\hline Categorías & Reconstrucción post terremoto & $\begin{array}{l}\text { Reconstrucción post incendios } \\
\text { forestales }\end{array}$ \\
\hline Principios orientadores & $\begin{array}{l}\text { Reposición rápida de viviendas. } \\
\text { Subsidiariedad. } \\
\text { Bajo control del proceso }\end{array}$ & $\begin{array}{l}\text { Reconstruir mejor. } \\
\text { Seguridad. } \\
\text { Calidad. } \\
\text { Intersectorialidad. } \\
\text { Participación. }\end{array}$ \\
\hline Enfoque de gestión & $\begin{array}{l}\text { Subsidio a la demanda. } \\
\text { Generación de valor social en lógica de ofer- } \\
\text { ta y demanda. } \\
\text { Mínimas modificaciones a los instrumentos } \\
\text { existentes. } \\
\text { Sesgo urbano. }\end{array}$ & $\begin{array}{l}\text { Flexibilización de los instrumentos } \\
\text { existentes. } \\
\text { Pertinencia. } \\
\text { Dirección pública para generar oferta y } \\
\text { orientación a la demanda }\end{array}$ \\
\hline Coordinación institucional & $\begin{array}{l}\text { No se levanta una institucionalidad ad hoc. } \\
\text { Enfoque centralista (escasa participación de } \\
\text { la estructura descentralizada en la toma de } \\
\text { decisiones). }\end{array}$ & $\begin{array}{l}\text { Coordinación Nacional. } \\
\text { Involucramiento de la estructura des- } \\
\text { centralizada de los ministerios. } \\
\text { Intersectorialidad (a nivel de autorida- } \\
\text { des, dificultades en mandos medios y } \\
\text { bajos) }\end{array}$ \\
\hline Planificación estatal & $\begin{array}{l}\text { Baja participación del Estado. } \\
\text { Responsabilidad en entes privados a través } \\
\text { de asociaciones formuladas en marcos insti- } \\
\text { tucionales débiles. } \\
\text { Impacto limitado de los planes (PRES) }\end{array}$ & $\begin{array}{l}\text { Organización multisectorial de arriba ha- } \\
\text { cia abajo, con participación comunitaria. } \\
\text { Alta participación del Estado. } \\
\text { Impacto alto del plan (PUIR). }\end{array}$ \\
\hline $\begin{array}{l}\text { Participación de actores } \\
\text { sociales y económicos }\end{array}$ & $\begin{array}{l}\text { Incentivo a la participación del sector priva- } \\
\text { do y organizaciones intermedias. } \\
\text { Escaso acompañamiento a los damnificados. }\end{array}$ & $\begin{array}{l}\text { Orientación a la economía local. } \\
\text { Fortalecimiento de la relación con } \\
\text { Municipalidades. } \\
\text { Participación comunitaria. } \\
\text { Complejidad en la relación con organiza- } \\
\text { ciones intermedias (fundaciones, ONG, } \\
\text { empresas). }\end{array}$ \\
\hline
\end{tabular}

Fuente: elaboración propia 
autores (Garcés, 2018), de que en Chile no hay continuidad en las políticas públicas relacionadas a los desastres, la mitigación de riesgos y la reconstrucción, indicando esto una gran dependencia de criterios políticos contingentes.

Evidentemente existen desafíos mayores en este sentido, que inclusive trascienden el ámbito de la gestión del riesgo y que, además, en todos los desastres socio-naturales se vuelven a presentar de manera puntual. En este sentido, un tema de fondo ineludible, tanto para el mundo público como para el empresarial y la sociedad civil -y que podría ser objeto de una próxima investigación- se refiere a la dimensión territorial de los desastres, que, en el caso de los incendios forestales, evidenció un problema de la sustentabilidad del modelo basado en la industria forestal.

\section{Referencias bibliográficas}

Bardin, L. (1996). El análisis de contenido. Barcelona: Akal

Barrera, D. (2017). Emergencia incendios forestales 2017. Chile: ODEPA. Recuperado de: https://www.odepa.gob.cl/wp-content/ uploads/2017/12/incendios2017.pdf

Cabello, A. (2015). Caracterización urbana de Talca: ciudad intermedia de Chile. Revista Nadir, $7(2), 1-22$.

Cárdenas, A. y Fuster, X. (2017). Desplazamientos en procesos de reconstrucción post catástrofe: El caso de Talca post-terremoto 27F. Revista AUS, (23), 34-41. doi:10.4206/aus.2018.n23-06

Comerio, M. (2015). Housing recovery lessons from Chile. Journal of the American Planning Association, 80(4), 340-350. doi:10.1080/01944363.2 014.968188

Concha, C., Micheletti, S., Olivares, R., y Rasse, A. (2015). Reconstrucción post-terremoto en la ruralidad del Maule. Bitácora Urbano Territorial, 25(01), 71-139. doi:10.15446/bitacora. $v \ln 25.40210$

Concha, C. y Rasse, A. (2014). La Ruta de las Caletas del Maule: Sobre la articulación entre sector público, privado y sociedad civil en los procesos de reconstrucción posterremoto. Revista de Geografía Norte Grande, (59), 165-184. doi:10.4067/S0718-34022014000300010 
Corporación Nacional Forestal. (2017a). Descripción y efectos "Tormenta de Fuego": 18 de enero al 5 de febrero de 2017. Regiones O'Higgins, El Maule y Biobio. [Diapositivas de PowerPoint]. Recuperado de https://www.conaf.cl/tormenta_de_fuego-2017/DESCRIPCION-Y-EFECTOS-TORMENTA-DE-FUEGO-18-ENEROAL-5-FEBRERO-2017.pdf

Corporación Nacional Forestal. (2017b). Análisis de la afectación y severidad de los incendios forestales: ocurridos en enero y febrero de 2017 sobre los usos de suelo y los ecosistemas naturales presentes entre las regiones de Coquimbo y Los Ríos de Chile. Informe Técnico. Recuperado de https:// www.conaf.cl/tormenta_de_fuego-2017/ INFORME-AFECTACION-Y_SEVERIDADDE-INCENDIOS-FORESTALES-VERANO2017-SOBRE-ECOSISTEMAS-VEGETACIONALES-CONAF.pdf

Corporación Nacional Forestal. (2019). Estadísticas - ocurrencia y daño por incendios forestales según incendios de magnitud 1985 - 2018. Recuperado de https://www.conaf.cl/incendios-forestales/incendios-forestales-en-chile/ estadisticas-historicas/

Delegación Presidencial para la Reconstrucción. (2014). Diagnóstico. Estado de la reconstrucción terremoto y tsunami 27 de febrero de 2010. Santiago de Chile: Ministerio del Interior y Seguridad Pública.

Delegación Presidencial para la Reconstrucción. (2015). Plan de cierre reconstrucción terremoto y tsunami 27F, 2010. Santiago de Chile: Ministerio del Interior y Seguridad Pública
Garcés, M. (2018). Lessons learned from Chile, evaluating strategic reconstruction master plans in post-disaster scenarios. (Tesis Master of Science in Urban Planning, sin publicar). Universidad de Columbia, Nueva York.

González, L. E. (2017). Gestión territorial post 27-F en Chile: implicancias sobre el hábitat residencial. Bitácora Urbano Territorial, 27(2), 109-116. doi:10.15446/bitacora.v27n2.48322

Herrmann, M. (2015). Urban planning and tsunami impact mitigation in Chile after February 27, 2010. Natural Hazards, 79(3), 1591-1620. doi:10.1007/s11069-015-1914-4

Imilan, W. y González, L. E. (2017). Attempts at neoliberal urban planning in postearthquake Chile Master Plans for reconstruction. Latin American Perspectives, 44(4), 10-23. doi:10.1177/0094582X16658265

Intveen, H. (2015). Intervenciones en paisajes urbanos residuales en el contexto del proceso de reconstrucción post 27f. Revista de Urbanismo, (32), 102-126. doi:10.5354/0717-5051.2015.36172

Inzulza, J. (2014). La recuperación del diseño cívico como reconstrucción de lo local en la ciudad intermedia: el caso de Talca, Chile. Revista AUS, (15), 4-8. doi:10.4206/aus.2014.n15-02

Inzulza, J. y Cárdenas, A. (2017). Desplazamiento subsidiario: efectos de gentrificación contemporánea en barrios céntricos en reconstrucción post-terremoto. El caso de Talca, Chile. Cuadernos Geográficos, 56(3), 268-291

Inzulza, J., Maragaño, A., Boano, C., y Díaz, I. (2018). Reconstrucción de ciudades intermedias en el siglo 
XXI - Procesos de gentrificación post desastres naturales. Talca: Universidad de Talca.

Irazábal, C. (2015). Learning from 27F: A comparative assessment of urban reconstruction processes after the 2010 eathquake in Chile. New York: Columbia University

Krippendorff, K. (1997). Metodología de análisis de contenido: Teoría y práctica. Barcelona: Paidós.

Letelier, F. y Boyco, P. (2011). Talca posterremoto: una ciudad en disputa. Modelo de reconstrucción, mercado inmobiliario y ciudadanía. Santiago de Chile: SUR

Letelier, F. y Rasse. A. (2016). Política de reconstrucción y desplazamiento: el caso de las familias de bajos ingresos del centro de Talca. Revista de Urbanismo, (35), 220-245. doi:10.5354/0717-5051.2016.42840

Micheletti, S. y Letelier, F. (2016). Damnificados de la reconstrucción post terremoto. Efectos del modelo en el hábitat rural del Maule. Revista INVI, 86(31), 106-122. doi:10.4067/ S0718-83582016000100002

Micheletti, S., Opazo, J., Rodríguez, C., Spuler, E., y Ríos, T. (2017). Relocalización pos terremoto. Trayectorias migratorias en damnificados rurales de la Región del Maule. Revista Pensamiento y Acción Disciplinaria, 3(1), 10-28.

MINVU. (2018). La reconstrucción en el marco de la gestión del riesgo de desastre. Documento de trabajo (no publicado).

ONU. (2015). Marco de Sendai para la reducción del riesgo de desastres 2015-2030. New York: Autor.
Onwuegbuzie, A., Dickinson, W., Leech, N., y Zoran, A. (2011). Un marco cualitativo para la recolección y análisis de datos en la investigación basada en grupos focales. Paradigmas, 3(2), 127-157.

Piñuel, J. (2002). Epistemología, metodología y técnicas del análisis de contenido. Sociolinguistic Studies, 3(1), 1-42. doi:10.1558/sols.v3il.1

Prieto, M. (2018). Infraestructura de lo común en tiempos de emergencia. Reconstrucción en Constitución y Llico. Revista INVI, 33(92), 125 194. doi:10.4067/S0718-83582018000100125 


\section{Anexo - Pautas de entrevista}

\section{PAUTA ENTREVISTA - AUTORIDADES Y FUNCIONARIOS PÚBLICOS}

La presente entrevista es parte de una investigación que pretende sistematizar el proceso de reconstrucción post incendios forestales en la región del Maule. La información obtenida será utilizada sólo con fines de investigación y de manera anónima, sin utilizar ningún tipo de información personal para dar cuenta de los resultados a menos que sea estrictamente necesario y usted nos dé su autorización explícita para tal fin. Asimismo, la entrevista es de carácter "voluntario", pudiendo retirarse de la misma en el momento que Ud. estime conveniente, no significándole ninguna responsabilidad posterior o consecuencia negativa.

1 ¿Qué cargo tenía durante el período del verano de 2017? ¿En qué consistía? ¿Qué rol cumplió el servicio / Ministerio donde se desempeñaba en la etapa de emergencia y reconstrucción?

2 ¿Recuerda cómo/cuando comenzaron los incendios? ¿Qué sucedió durante esos días?

3 Considerando que en Chile frecuentemente ocurren desastres naturales, ¿Cómo se encontraba preparado el país para una situación como esta? ¿Con qué mecanismos se contaba para enfrentar la emergencia?
4 ¿Cuánto y cómo influyó el actual modelo de uso del territorio (plantaciones forestales) en el desarrollo del desastre? ¿En términos sociales, la presencia de plantaciones forestales en el Secano Interior de la Región representa un beneficio para las comunidades o aumenta su vulnerabilidad?

5 ¿Qué acciones se llevaron a cabo en la institución a la cual usted pertenece (hitos)?

6 iQuiénes cumplieron un rol fundamental en el periodo de la emergencia? ¿Cómo funcionó el trabajo coordinado con otras instituciones/servicios/ministerios?

7 ¿Cómo evalúa la respuesta del Estado frente a la emergencia? ¿Qué hechos marcaron ese periodo?

8 ¿Cómo se trabajó en los sectores apartados?

9 ¿En qué consistió la ayuda entregada a las personas afectadas por los incendios forestales?

10 Un aspecto fundamental en los tratados internacionales referidos a los desastres socionaturales es la protección de derechos de las personas afectadas ¿Cómo siente que se abordó el tema por parte de los actores involucrados durante la emergencia?

11 ¿Cómo se resguarda el derecho a la educación y el derecho al trabajo en situaciones como la ocurrida?

12 ¿Cómo se abordó el tema de la salud física y mental de las personas involucradas en el proceso (damnificados/voluntarios/funcionarios)? 
13 ¿En qué momento sienten que la emergencia ha terminado? ¿Cómo se desarrolla el período posterior a la emergencia?

14 En el caso de la reconstrucción que se está llevando a cabo, ¿Qué opina sobre el marco de la política de vivienda orientado al hábitat rural?

15 ¿Quiénes han estado presente a lo largo del proceso de reconstrucción (personas/instituciones)? ¿Mediante qué acciones? ¿Cómo evalúa su intervención?

16 iSe establecieron mecanismos de participación y consulta con los afectados? ¿Cuales? ¿Cómo funcionaban? ¿De qué modo se tomaron en consideración las opiniones de las personas?

17 ¿Se establecieron mecanismos de participación y consulta con otros actores interesados en la reconstrucción? ¿Cuales? ¿Cómo funcionaban?

18 Me gustaría volver sobre el punto de los derechos de las personas afectadas por los incendios, pero esta vez centrándonos en el proceso de reconstrucción, ¿Cómo siente que se está abordando el tema por parte de los actores involucrados?

19 Entendiendo que las características y las condiciones en las que ocurrieron son diferentes pero que, sin embargo, es posible establecer ciertos puntos en común iqué diferencias ve con respecto a lo ocurrido durante el 27F? ¿Qué aprendizajes del 27F sirvieron para enfrentar esta emergencia?
20 ¿Cómo evalúa el proceso de reconstrucción hasta la fecha? ¿Qué desafíos quedan pendientes?

21 ¿Cuáles son los aprendizajes que quedan para su (ex) institución luego del desastre, en el ámbito de la emergencia y reconstrucción?

Para el cierre de la entrevista se recomienda agradecer la disposición del entrevistado y ofrecerle un espacio para que pueda agregar información que crea necesaria.

\section{PAUTA DE ENTREVISTA - ACTORES VINCULADOS (TERCER SECTOR, ACADEMIA, EMPRESA)}

La presente entrevista es parte de una investigación que pretende sistematizar el proceso de reconstrucción post incendios forestales en la región del Maule. La información obtenida será de utilizada sólo con fines de investigación y de manera anónima, sin utilizar ningún tipo de información personal para dar cuenta de los resultados a menos a menos que sea estrictamente necesario y usted nos dé su autorización explícita para tal fin. Asimismo, la entrevista es de carácter "voluntario", pudiendo retirarse de la misma en el momento que Ud. estime conveniente, no significándole ninguna responsabilidad posterior o consecuencia negativa.

1 Nos gustaría comenzar conociéndolo un poco, ¿Cuál es su nombre? ¿A qué organización pertenece y a que se dedica? ¿Se 
encontraba en el mismo cargo durante los incendios del 2017 ?

2 ¿Tenía alguna relación con las localidades afectadas previo a la emergencia? ¿De qué tipo?

3 ¿Recuerda cómo/cuando comenzaron los incendios? ¿Qué sucedió durante esos días?

4 Durante la emergencia, ¿Qué acciones llevaron a cabo en la institución a la cual usted pertenece?

5 ¿Cómo funcionó el trabajo coordinado con otros actores institucionales?

6 ¿Qué hechos marcaron el periodo de emergencia?

7 ¿Las estrategias de acción fueron focalizadas en un territorio específico? ¿Se utilizaron estrategias de acción para los territorios rurales más alejados? ¿Cuales?

8 ¿En qué consistió la ayuda entregada a las personas afectadas por los incendios forestales?

9 Un aspecto fundamental en los tratados internacionales referidos a los desastres socionaturales es la protección de derechos de las personas afectadas ¿Cómo siente que se abordó el tema por parte de los actores involucrados durante la emergencia?

10 Dependiendo del entrevistado ${ }^{13}$ : ¿Cómo se resguarda el derecho a la educación y el

13 Las preguntas marcadas como Dependiendo del entrevistado se realizan solo en aquellos casos que resulten pertinentes y cuando el actor involucrado tenga alguna relación con la temática de la pregunta. derecho al trabajo en situaciones como la ocurrida?

11 ¿Cómo se resguardó el tema de la salud física y mental de las personas involucradas en el proceso (damnificados/voluntarios/ funcionarios)?

12 ¿En qué momento sienten que la emergencia ha terminado? ¿Cómo se desarrolla el período posterior a la emergencia?

13 ¿Qué otros actores han estado presentes a lo largo del proceso de reconstrucción (personas/instituciones)? ¿Mediante qué acciones? ¿Cómo evalúa su intervención? ¿Existió algún tipo de coordinación con ellos?

14 ¿Se establecieron mecanismos de participación y consulta entre los actores involucrados? ¿¿Cuales? ¿Cómo funcionaban?

15 Me gustaría volver sobre el punto de los derechos de las personas afectadas por los incendios, pero esta vez centrándonos en el proceso de reconstrucción, ¿Cómo siente que se está abordando el tema por parte de los actores involucrados?

16 Entendiendo que las características y las condiciones en las que ocurrieron son diferentes pero que sin embargo es posible establecer ciertos puntos en común iqué diferencias ve con respecto a lo ocurrido durante el 27F? ¿Qué aprendizajes del 27F sirvieron para enfrentar esta emergencia? 
17 ¿Cómo evalúa el proceso de reconstrucción hasta la fecha? ¿Qué desafíos quedan pendientes?

Para el cierre de la entrevista se recomienda agradecer la disposición del entrevistado y ofrecerle un espacio para que pueda agregar información que crea necesaria.

\section{PAUTA FOCUS GROUP}

El presente focus group es parte de una investigación que pretende sistematizar el proceso de reconstrucción post incendios forestales en la región del Maule. La información obtenida será utilizada sólo con fines de investigación y de manera anónima, sin utilizar ningún tipo de información personal para dar cuenta de los resultados a menos que sea estrictamente necesario y usted nos dé su autorización explícita para tal fin. Asimismo, el focus group es de carácter "voluntario", pudiendo retirarse del mismo en el momento que Ud. estime conveniente, no significándole ninguna responsabilidad posterior o consecuencia negativa.

\section{Preguntas guía}

1 Presentación de cada participante de la actividad: Nombre, rol que cumplían en la institución/organización al momento de la emergencia y/o reconstrucción.

2 ¿Recuerdan cómo/cuando comenzaron los incendios? ¿Qué sucedió durante esos días?
3 ¿En qué consistió el trabajo que ustedes y la institución a la que pertenecen realizaron durante los incendios forestales?

4 ¿Qué actores (además de ustedes) identifican como relevantes al momento de la emergencia y de la reconstrucción?

5 Un aspecto fundamental en los tratados internacionales referidos a los desastres socionaturales es la protección de derechos de las personas afectadas ¿Cómo sienten que se abordó el tema por parte de los actores involucrados durante la emergencia y la reconstrucción?

6 ¿En qué momento sienten que la emergencia ha terminado? ¿Cómo se desarrolla el período posterior a la emergencia?

7 ¿Se establecieron mecanismos de participación, consulta y coordinación entre los actores involucrados? ¿Cuales? ¿Cómo funcionaban?

8 Entendiendo que las características y las condiciones en las que ocurrieron son diferentes pero que, sin embargo, es posible establecer ciertos puntos en común, ¿qué diferencias ve con respecto a lo ocurrido durante el 27F? ¿Qué aprendizajes del 27F sirvieron para enfrentar esta emergencia?

9 ¿Cómo evalúan el proceso de reconstrucción hasta la fecha? ¿Qué desafíos quedan pendientes? 


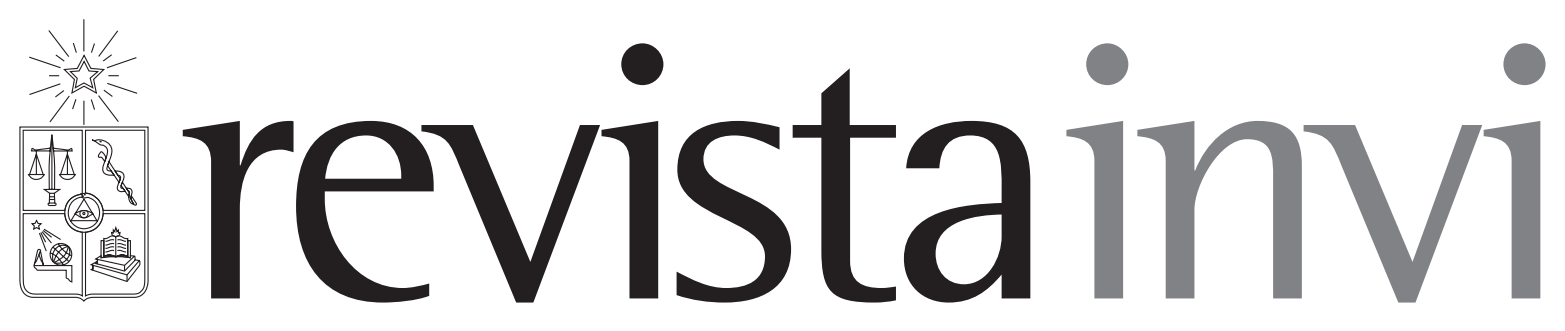

Revista INVI es una publicación periódica, editada por el Instituto de la Vivienda de la Facultad de Arquitectura y Urbanismo de la Universidad de Chile, creada en 1986 con el nombre de Boletín INVI. Es una revista académica con cobertura internacional que difunde los avances en el conocimiento sobre la vivienda, el hábitat residencial, los modos de vida y los estudios territoriales. Revista INVI publica contribuciones originales en español, inglés y portugués, privilegiando aquellas que proponen enfoques inter y multidisciplinares y que son resultado de investigaciones con financiamiento y patrocinio institucional. Se busca, con ello, contribuir al desarrollo del conocimiento científico sobre la vivienda, el hábitat y el territorio y aportar al debate público con publicaciones del más alto nivel académico.

Director: Dr. Ricardo Tapia Zarricueta, Universidad de Chile, Chile.

Editor: Dr. Luis Campos Medina, Universidad de Chile, Chile.

Editor asistente: Dr. Walter Imilan, Universidad de Chile, Chile.

Coeditora: Srta. Sandra Rivera, Universidad de Chile, Chile.

\section{COMITÉ EDITORIAL:}

Dr. Victor Delgadillo, Universidad Autónoma de la Ciudad de México, México.

Dra. María Mercedes Di Virgilio, CONICET/ IIGG, Universidad de Buenos Aires, Argentina.

Dra. Irene Molina, Uppsala Universitet, Suecia.

Dr. Gonzalo Lautaro Ojeda Ledesma, Universidad de Valparaíso, Chile.

Dra. Suzana Pasternak, Universidade de São Paulo, Brasil.

Dr. Javier Ruiz Sánchez, Universidad Politécnica de Madrid, España.

Dra. Elke Schlack Fuhrmann, Pontificia Universidad Católica de Chile, Chile.

Dr. Carlos Alberto Torres Tovar, Universidad Nacional de Colombia, Colombia.

Sitio web: http://www.revistainvi.uchile.cl/

Correo electrónico: revistainvi@uchilefau.cl

Licencia de este artículo: Creative Commons Atribución-Compartirlgual 4.0 Internacional (CC BY-SA 4.0) 